\title{
Thruster and Vibration Control of Marine Powertrain Using a Class of Feedforward Approximators
}

\author{
Pey Yuen Tao, Shuzhi Sam Ge*, Tong Heng Lee and Xiaoqi Chen
}

\begin{abstract}
In this paper, we consider the tracking problem of propeller shaft speed and simultaneously minimizing torsional vibrations in marine shafting system, in the presence of parametric/functional uncertainties and unmodelled dynamics. Torsional vibrations within the shafting system can be induced by the hydrodynamic forces acting on the propeller and the inertia forces of the crank mechanism. Excessive vibrations will lead to severe consequences such as fractured drive shaft and compromised structural integrity. Due to the difficulty in measuring or modelling the hydrodynamic forces as well as the frictional forces, neural networks are used to compensate for the uncertainties. Simulation results illustrate the effectiveness of the proposed controller.
\end{abstract}

\section{INTRODUCTION}

The problem of torsional vibrations within the marine shafting system, in the presence of parametric and functional uncertainties, poses a challenge to both marine engineering practitioners and control theorists alike. Torsional vibrations within the propulsive shafting system of a marine vessel can be induced by the hydrodynamic forces acting on the propeller and inertia forces of the crank mechanism. Excessive torsional vibrations within the shafting system will lead to failure of the drive shaft. Furthermore, the propulsion system is connected to the ship's hull which facilitates the transfer of vibrational energy within the shafting system to other sections of the vessel leading to excessive noise and compromising the structural integrity. A common approach to tackle the problem of excessive vibrations is to design the shafting system such that vibration response is within the allowable limits. Several methods have been proposed for the modelling of a marine shafting system [1] [2]. In this paper, we consider the method presented in [3] wherein the marine shafting system is modelled as a chained multiple mass-spring-damper system.

The main focus in torsional vibration suppression within marine shafting systems has been directed towards the design of the shafting system. The design of an effective control strategy to actively minimize torsional vibrations in marine shafting systems during operation have received relatively little attention. In general, the main objective of marine propulsion control focuses on achieving the desired position and velocity for the marine vessel through the control of shaft speed for fixed pitch (FP) propellers and pitch for

Pey Yuen Tao, Shuzhi Sam Ge and Tong Heng Lee are with Department of Electrical \& Computer Engineering, National University of Singapore, Singapore 119260

Xiaogi Chen is with Singapore Institute of Manufacturing Technology, 71 Nanyang Drive, Singapore 638075

* To whom all correspondences should be addressed. Tel: (+65) 6874 6821, Fax: (+65) 6779 1103, E-mail: elegesz@nus.edu.sg controllable pitch (CP) propellers. Through the estimation of the propeller axial flow velocity, a nonlinear output feedback controller was developed in [4] for underwater vehicles where varying propeller thrust due to unsteady flow effects were compensated. Recent developments in marine propulsion control focus on the control of the power from the drive system to achieve the desired thrust [5] which achieves improved performance in moderate seas over conventional shaft speed controller.

Hydrodynamic forces acting on the propeller are highly nonlinear and subjected to variations due to the diverse operating conditions such as air suction, cavitation and partial/full emergence of the propellers [4]- [6]. Furthermore, torsional stiffness of the drive shaft will be subjected to changes during operation due to the mechanical wear and tear of the shafting system. Traditional adaptive controllers are generally useful when dealing with systems whose dynamics can be expressed in the linear-in-parameters form, for which the regressor is exactly known and the uncertainty is parametric and time-invariant. Such a restriction is clearly ill-suited for this system. To overcome the limitations of model-based adaptive controllers, we adopt approximation-based control techniques to compensate for functional uncertainties in the dynamic model of the shafting system. According to the Stone-Weierstrass theorem [7], a universal approximator can approximate, to an arbitrary degree of accuracy, any real continuous function on a compact set. Such approximators can utilize a standard regressor function whose structure is independent of the dynamic characteristics of the forces acting on the shafting system.

Motivated by results in control of flexible joint robots [8][10], we propose a Lyapunov-based approximation-based controller for the control of the marine shafting system. The control objective is to track a desired trajectory and simultaneously minimize the torsional vibrations within the shafting system. The main contributions of this paper are:

(i) the use of Radial Basis Functions (RBF) Neural Networks (NN) to compensate for the parametric and functional uncertainties which are commonly faced in thruster control. Rigorous stability analysis shows that semiglobal uniform boundedness of the tracking error is guaranteed, and the residual error can be made arbitrarily small by appropriate choice of the design parameters;

(ii) the investigation of torsional vibrations within the shafting system and the design of an active controller to reduce the vibrations. 
The organization of the remainder of this paper is as follows. Section Il introduces some technical preliminaries required in the sequel, the dynamic model of the system and the structure of the neural networks (NN) used in the controller design. Thereafter, we present, in Section III, the adaptive NN control design procedure for marine shafting system under unknown hydrodynamic and frictional forces. A simulation study is conducted to investigate the effectiveness of the proposed control design and the results are illustrated in Section IV. Finally, the conclusions are given in Section V.

\section{Mathematical Preliminaries}

In this section, we present some notions and assumptions that will be used in the subsequent developments.

Lemma 2.1: For bounded initial conditions, if there exists a $C^{1}$ continuous and positive definite Lyapunov function $V(x)$ satisfying $\kappa_{1}(\|x\|) \leq V(x) \leq \kappa_{2}(\|x\|)$, such that $\dot{V}(x) \leq-\rho V(x)+c$, where $\kappa_{1}, \kappa_{2}: R^{n} \rightarrow R$ are class $\mathrm{k}$ functions and $c$ is a positive constant, then the solution $x=0$ is uniformly bounded [11].

Assumption 2.1: The desired propeller trajectory, $q_{d}(t)$, is a bounded $C^{2 n+2}$ function.

\section{A. Dynamic Modelling}

From the results presented in [3], the marine shafting system can be effectively modelled as a chained multiple massspring-damper system. In this paper, the controller is developed utilizing the following chained multiple mass-springdamper model of the marine shafting system:

$$
\begin{aligned}
I \ddot{q} & =k\left(\theta_{1}-q\right)+f(q, \dot{q}) \\
I_{1} \ddot{\theta}_{1} & =k_{1}\left(\theta_{2}-\theta_{1}\right)-k\left(\theta_{1}-q\right)+f_{1}\left(\theta_{1}, \dot{\theta}_{1}\right) \\
& \cdots \\
I_{i} \ddot{\theta}_{i} & =k_{i}\left(\theta_{i+1}-\theta_{i}\right)-k_{i-1}\left(\theta_{i}-\theta_{i-1}\right)+f_{i}\left(\theta_{i}, \dot{\theta}_{i}\right) \\
& \cdots \\
I_{n} \ddot{\theta}_{n} & =u-k_{n-1}\left(\theta_{n}-\theta_{n-1}\right)+f_{n}\left(\theta_{n}, \dot{\theta}_{n}\right)
\end{aligned}
$$

where $k_{i}$ is the unknown torsional stiffness of the massless spring connecting the $i$ th mass unit to the $(i+1)$ th mass unit, $I_{i}$ is the unknown moment of inertia of the $i$ th mass unit about the rotating axis, $f$ is a function of unknown hydrodynamic forces acting on the propeller, $f_{i}$ is a function of unknown frictional forces acting on the $i$ th unit and $u$ is the torque produced by the motor. In this paper, we consider the motor torque as the control input to the system.

\section{B. Feedforward Neural Networks}

Functional approximators can be represented as multi-layer feedforward networks which may be nonlinearly- or linearlyparameterized. A class of linearly parameterized feedforward approximators used to approximate the continuous function $f(Z): R^{q} \rightarrow R$ may be represented as follows:

$$
f_{n n}(Z)=W^{T} S(Z)
$$

where the vector $Z=\left[z_{1}, \cdots, z_{q}\right]^{T} \in R^{q}$ are the input variables to the approximator, $W \in R^{l}$ is a vector of adaptable weights and $S(Z)=\left[s_{1}(Z), \cdots, s_{l}(Z)\right]^{T} \in R^{l}$ is a vector of known continuous (linear or nonlinear) basis functions, with $s_{i}(Z)$ being chosen as the commonly used Gaussian functions,

$s_{i}(Z)=\exp \left[\frac{-\left(Z-\mu_{i}\right)^{T}\left(Z-\mu_{i}\right)}{\eta_{i}^{2}}\right], \quad i=1, \cdots, l$

where $\mu_{i}=\left[\mu_{i 1}, \mu_{i 2}, \cdots, \mu_{i q}\right]^{T}$ is the center of the receptive field for node $i$ and $\eta_{i}$ is the width of the Gaussian function. Universal approximation results in [7] indicate that if $l$ is chosen sufficiently large, $W^{T} S(Z)$ can approximate any continuous function, $f(Z)$, to any desired accuracy over a compact set $\Omega_{Z} \subset R^{q}$ to any arbitrary degree of accuracy.

$$
f(Z)=W^{* T} S(Z)+\varepsilon(Z), \quad \forall Z \in \Omega_{Z} \subset R^{q}
$$

where $W^{*}$ is the ideal constant weight vector and $\varepsilon(Z)$ is the approximation error for the special case where $W=W^{*}$ which is bounded over the compact set, i.e., $|\varepsilon(Z)| \leq \bar{\varepsilon}$, $\forall Z \in \Omega_{Z}$ where $\bar{\varepsilon}>0$ is an unknown constant. The ideal weight vector $W^{*}$, an artificial quantity required for analytical purposes, is defined as the value of $W$ that minimizes $|\varepsilon(Z)|$ for all $Z \in \Omega_{Z} \subset R^{q}$, i.e.,

$$
W^{*}:=\arg \min _{W \in R^{l}}\left\{\sup _{Z \in \Omega_{Z}}\left|f(Z)-W^{T} S(Z)\right|\right\}
$$

\section{iII. Controller Design}

In this section, a controller is developed utilizing neural networks and backstepping where that the individual torsional displacement and velocities, $q, \dot{q}, \theta_{i}$ and $\dot{\theta}_{i}$, are measurable. Step 1: First, we introduce a virtual control, $\alpha_{r}$, and a perturbation $z_{1}=\theta_{1}-\alpha_{r}$ into (1), we have

$$
I \ddot{q}+k q=f+k \alpha_{r}+k z_{1}
$$

A chain of backstepping is required until the physical control input appears in the error equations. The objective is to make $z_{1}$ small so that the propeller dynamics can be controlled for tracking purposes. The derivative of $z_{1}$ is given by $\dot{z}_{1}=$ $\dot{\theta}_{1}-\dot{\alpha}_{r}$. A virtual control input, $\alpha_{1}$, is introduced to drive $z_{1}$ to a small value as described by

$$
\dot{z}_{1}=z_{2}+\alpha_{1}-\dot{\alpha}_{r}
$$

where $z_{2}=\dot{\theta}_{1}-\alpha_{1}$ is the second perturbation term to be controlled. The derivative of $z_{2}$ is given by

$$
I_{1} \dot{z}_{2}=k_{1}\left(\theta_{2}-\theta_{1}\right)+f_{1}\left(\theta_{1}, \dot{\theta}_{1}\right)-I_{1} \dot{\alpha}_{1}
$$

A virtual control input, $\alpha_{2}$, is introduced to drive $z_{2}$ to a small value as described by

$$
I_{1} \dot{z}_{2}=k_{1} \alpha_{2}-k_{1} \theta_{1}+f_{1}\left(\theta_{1}, \dot{\theta}_{1}\right)-I_{1} \dot{\alpha}_{1}+k_{1} z_{3}
$$

where $z_{3}=\theta_{2}-\alpha_{2}$.

Step $i$ : Applying the above methods recursively for each step,

$$
z_{2 i-1}=\theta_{i}-\alpha_{2 i-2}
$$

where $\alpha_{2 i-2}$ is the virtual control input to stabilize the $i-1$ th subsystem and the control objective for the $i$ th step is to-drive $z_{2 i-1}$ to a small value. Introducing a virtual control input, 
$\alpha_{2 i-1}$, to drive $z_{2 i-1}$ to a small value, the derivative of $z_{2 i-1}$ is given by

$$
\dot{z}_{2 i-1}=z_{2 i}+\alpha_{2 i-1}-\dot{\alpha}_{2 i-2}
$$

where $\hat{z}_{2 i}=\dot{\theta}_{i}-\alpha_{2 i-1}$ is a perturbation term to be controlled. The derivative of $z_{2 i}$ is given by

$$
\begin{aligned}
I_{i} \dot{\bar{z}}_{2 i}= & k_{i}\left(\theta_{i+1}-\theta_{i}\right)-k_{i-1}\left(\theta_{i}-\theta_{i-1}\right) \\
& +f_{i}\left(\theta_{i}, \dot{\theta}_{i}\right)-I_{i} \dot{\alpha}_{2 i-1}
\end{aligned}
$$

A virtual control input, $\alpha_{2 i}$, is introduced to drive $z_{2 i}$ to a small value as described by

$$
\begin{aligned}
I_{i} \dot{z}_{2 i}= & k_{i} \alpha_{2 i}-k_{i} \theta_{i}-k_{i-1}\left(\theta_{i}-\theta_{i-1}\right) \\
& +f_{i}\left(\theta_{i}, \dot{\theta}_{i}\right)-I_{i} \dot{\alpha}_{2 i-1}+k_{i} z_{2 i+1}
\end{aligned}
$$

where $z_{2 i+1}=\theta_{i+1}-\alpha_{2 i}$.

Step $n$ : For the last step, we have

$$
z_{2 n-1}=\theta_{n}-\alpha_{2 n-2}
$$

where $\alpha_{2 n-2}$ is the virtual control input to stabilize the $n-$ 1 th subsystem and the control objective for the $n$th step is to drive $z_{2 n-1}$ to a small value. Introducing a virtual control input, $\alpha_{2 n-1}$, to drive $z_{2 n-1}$ to a small value, the derivative of $z_{2 n-1}$ is given by

$$
\dot{z}_{2 n-1}=z_{2 n}+\alpha_{2 n-1}-\dot{\alpha}_{2 n-2}
$$

where $z_{2 n}=\dot{\theta}_{n}-\alpha_{2 n-1}$ is a perturbation term to be controlled. The derivative of $z_{2 n}$ is given by

$$
I_{n} \dot{z}_{2 n}=u-k_{n}\left(\theta_{n}-\theta_{n-1}\right)+f_{n}\left(\theta_{n}, \dot{\theta}_{n}\right)-I_{n} \dot{\alpha}_{2 n-1}
$$

The controller design now becomes the design of $\alpha_{r}$ in (2), $\alpha_{2 i-1}$ in (3), $\alpha_{2 i}$ in (4) and $u$ in (5). First, we define the desired trajectory for the propeller as $q_{d}$, where $q_{d}(t)$ is a $C^{2 n+2}$ function. Define the tracking error as

$$
e(t)=q_{d}(t)-q(t)
$$

where the control objective is the tracking of the desired trajectory $q_{d}(t)$. A dynamic compensator is defined as

$$
r=\dot{e}+\lambda e
$$

where $\lambda$ is a design parameter. Using the above equations and (2), we obtain

$$
I \dot{r}=I\left(\ddot{q}_{d}+\lambda \dot{e}\right)+k q-f-k \alpha_{r}-k z_{1}
$$

Consider the following control laws

$$
\begin{aligned}
\alpha_{r}= & \frac{1}{k}\left(\kappa_{r} r+I\left(\ddot{q}_{d}+\lambda \dot{e}\right)-f\right)+q \\
\alpha_{2 i-1}= & -\kappa_{2 i-1} z_{2 i-1}+\dot{\alpha}_{2 i-2}-z_{2 i-2} \\
\alpha_{2 i}= & \frac{1}{k_{i}}\left(-\kappa_{2 i} z_{2 i}+k_{i-1}\left(\theta_{i}-\theta_{i-1}\right)\right. \\
-\quad & \left.+I_{i} \dot{\alpha}_{2 i-1}-f_{i}\right)-z_{2 i-1}+\theta_{i} \\
u= & -\kappa_{2 n} z_{2 n}+k_{n}\left(\theta_{n}-\theta_{n-1}\right)-f_{n} \\
& -z_{2 n-1}+I_{n} \dot{\alpha}_{2 n-1}
\end{aligned}
$$

where $\kappa_{i}$ are design parameters. The above control laws cannot be realized as the torsional stiffness $k_{i}$, inertia $I_{i}$ and the functions of frictional and hydrodynamic forces $f_{i}$ are unknown. Therefore, the time derivatives of the virtual control

$$
\begin{aligned}
\dot{\alpha}_{r}= & \frac{\partial \alpha_{r}}{\partial q} \dot{q}+\frac{\partial \alpha_{r}}{\partial \dot{q}} \ddot{q}+\sum_{j=1}^{3} \frac{\partial \alpha_{r}}{\partial q_{d}^{(j-1)}} q_{d}^{(j)} \\
\dot{\alpha}_{2 i-1}= & \frac{\partial \alpha_{2 i-1}}{\partial q} \dot{q}+\frac{\partial \alpha_{2 i-1}}{\partial \dot{q}} \ddot{q}+\sum_{j=1}^{i} \frac{\partial \alpha_{2 i-1}}{\partial \theta_{j}} \dot{\theta}_{j} \\
& +\sum_{j=1}^{i-1} \frac{\partial \alpha_{2 i-1}}{\partial \dot{\theta}_{j}} \ddot{\theta}_{j}+\sum_{j=1}^{2 i+2} \frac{\partial \alpha_{2 i-1}}{\partial q_{d}^{(j-1)}} q_{d}^{(j)} \\
\dot{\alpha}_{2 i}= & \frac{\partial \alpha_{2 i}}{\partial q} \dot{q}+\frac{\partial \alpha_{2 i}}{\partial \dot{q}} \ddot{q}+\sum_{j=1}^{i}\left(\frac{\partial \alpha_{2 i}}{\partial \theta_{j}} \dot{\theta}_{j}+\frac{\partial \alpha_{2 i}}{\partial \dot{\theta}_{j}} \ddot{\theta}_{j}\right) \\
& +\sum_{j=1}^{2 i+3} \frac{\partial \alpha_{2 i}}{\partial q_{d}^{(j-1)}} q_{d}^{(j)}
\end{aligned}
$$

contains unknown terms as well. Define $h_{i}$ as a function of unknown terms and $\phi_{i}$ as a function of known terms in $\dot{\alpha}_{i}$. By employing feedforward approximators to approximate the unknown functions where

$$
\begin{aligned}
W_{r}^{*^{T}} S_{r}= & \frac{1}{k}\left(f-I\left(\ddot{q}_{d}+\lambda \dot{e}\right)\right)-\varepsilon_{r} \\
W_{2 i-1}^{*^{T}} S_{2 i-1}= & \frac{\partial \alpha_{2 i-2}}{\partial \dot{q}} \ddot{q}+\sum_{j=1}^{i-1} \frac{\partial \alpha_{2 i-2}}{\partial \dot{\theta}_{j}} \ddot{\theta}_{j}-\varepsilon_{2 i-1} \\
W_{2 i}^{*^{T}} S_{2 i}= & \frac{1}{k_{i}}\left(\frac{\partial \alpha_{2 i-1}}{\partial \dot{q}} \ddot{q}+f_{i}-k_{i-1}\left(\theta_{i}-\theta_{i-1}\right)\right. \\
& \left.-I_{i} \phi_{2 i-1}+\sum_{j=1}^{i-1} \frac{\partial \alpha_{2 i-1}}{\partial \dot{\theta}_{j}} \ddot{\theta}_{j}\right)-\varepsilon_{2 i} \\
W_{2 n}^{*^{T}} S_{2 n}= & \frac{\partial \alpha_{2 n-1}}{\partial \dot{q}} \ddot{q}+\sum_{j=1}^{n-1} \frac{\partial \alpha_{2 n-1}}{\partial \dot{\theta}_{j}} \ddot{\theta}_{j}+f_{n} \\
& -k_{n-1}\left(\theta_{n}-\theta_{n-1}\right)-I_{n} \phi_{2 n-1}-\varepsilon_{2 i}
\end{aligned}
$$

Thus, the practical approximation-based controllers can be realized as follows:

$$
\begin{aligned}
\alpha_{r}= & \left(\kappa_{r}+\kappa_{W r}\right) r-\hat{W}_{r}^{T} S_{r}\left(Z_{r}\right)+q \\
\alpha_{1}= & -\left(\kappa_{1}+\kappa_{W 1}\right) z_{1}+r-\hat{W}_{1}^{T} S_{1}\left(Z_{1}\right)+\phi_{r} \\
\alpha_{2 i-1}= & -\left(\kappa_{2 i-1}+\kappa_{W 2 i-1}\right) z_{2 i-1}-z_{2 i-2} \\
& -\hat{W}_{2 i-1}^{T} S_{2 i-1}\left(Z_{2 i-1}\right)+\phi_{2 i-2} \\
\alpha_{2 i}= & -\left(\kappa_{2 i}+\kappa_{W 2 i}\right) z_{2 i}-\hat{W}_{2 i}^{T} S_{2 i}\left(Z_{2 i}\right)-z_{2 i-1}+\theta_{i} \\
u= & -\left(\kappa_{2 n}+\kappa_{W 2 n}\right) z_{2 n}-\hat{W}_{2 n}^{T} S_{2 n}\left(Z_{2 n}\right) \\
& -z_{2 n-1}
\end{aligned}
$$

where $\hat{W}_{i}$ approximates $W_{i}^{*}$.

Remark 3.1: Although the terms $\frac{\partial \alpha_{i}}{\partial \dot{q}}$ and $\frac{\partial \alpha_{i}}{\partial \dot{\theta}_{k}}$ contain the neural networks weights $\hat{W}_{j}$ for $j=1,2, \ldots, i$, the large number of neural network weight estimates $\hat{W}_{j}$ are not recommended to be taken as inputs to the $\mathrm{NN}$ because of the curse of dimensionality of RBF NN [12] [13]. 
By defining intermediate variables $\frac{\partial \alpha_{i}}{\partial \dot{q}}$ and $\frac{\partial \alpha_{i}}{\partial \dot{\theta}_{k}}$ which are available through computation, the $\mathrm{NN}$ approximation $\hat{W}_{i}^{T} S_{i}\left(Z_{i}\right)$ of the unknown terms can be computed using the minimal number of $\mathrm{NN}$ inputs where

$$
\begin{aligned}
Z_{r} & =\left[\ddot{q}_{d}+\lambda \dot{e}, q, \dot{q}\right]^{T} \\
Z_{2 i-1} & =\left[q, \dot{q}, \bar{\theta}_{i}, \overline{\dot{\theta}}_{i-1}, \frac{\partial \alpha_{2 i-2}}{\partial \dot{q}}, \frac{\partial \alpha_{2 i-2}}{\partial \dot{\theta}_{1}}, \ldots, \frac{\partial \alpha_{2 i-2}}{\partial \dot{\theta}_{i-1}}\right]^{T} \\
Z_{2 i} & =\left[q, \dot{q}, \bar{\theta}_{i}, \overline{\dot{\theta}}_{i}, \frac{\partial \alpha_{2 i-1}}{\partial \dot{q}}, \frac{\partial \alpha_{2 i-1}}{\partial \dot{\theta}_{1}}, \ldots, \frac{\partial \alpha_{2 i-1}}{\partial \dot{\theta}_{i-1}}, \phi_{2 i-1}\right]^{T} \\
Z_{2 n} & =\left[q, \dot{q}, \theta, \dot{\theta}, \frac{\partial \alpha_{2 n-1}}{\partial \dot{q}}, \frac{\partial \alpha_{2 n-1}}{\partial \dot{\theta}_{1}}, \ldots, \frac{\partial \alpha_{2 n-1}}{\partial \dot{\theta}_{n-1}}, \phi_{2 n-1}\right]^{T}
\end{aligned}
$$

Consider the following Lyapunov function candidate

$$
V=\frac{1}{2} z^{T} M z+\frac{1}{2} \bar{W}_{r}^{T} \Gamma_{r}^{-1} \tilde{W}_{r}+\sum_{i=1}^{2 n} \frac{1}{2} \tilde{W}_{i}^{T} \Gamma_{i}^{-1} \tilde{W}_{i}
$$

where $z=\left[r, z_{1}, z_{2}, \ldots, z_{2 n}\right]^{T}, \Gamma_{i}$ are constant gain matrices, $\bar{W}_{i}=\hat{W}_{i}-W_{i}^{*}$ and $M \in R^{2 n+1 \times 2 n+1}$ is a diagonal matrix with $\frac{I}{k}$ as its first diagonal element, 1 and $\frac{I_{i}}{k_{i}}$ as its $(2 i)$ th and $(2 i+1)$ th diagonal element respectively. The time derivative of $V$ is given by

$$
\dot{V}=z^{T} M \dot{z}+\tilde{W}_{r}^{T} \Gamma_{r}^{-1} \dot{\hat{W}}_{r}+\sum_{i=1}^{2 n} \tilde{W}_{i}^{T} \Gamma_{i}^{-1} \dot{\hat{W}}_{i}
$$

Substituting the virtual controllers, $\alpha_{i}$, and control input, $u$, into (8), we obtain

$$
\begin{aligned}
\dot{V}= & -\hat{z}^{T}\left(K+K_{w}\right) z+\tilde{W}_{r}^{T}\left(\Gamma_{r}^{-1} \dot{\hat{W}}_{r}+S_{r}\left(Z_{r}\right) r\right) \\
& +\sum_{i=1}^{2 n} \tilde{W}_{i}^{T}\left(\Gamma_{i}^{-1} \dot{\hat{W}}_{i}-S_{i}\left(Z_{i}\right) z_{i}\right)+\varepsilon z
\end{aligned}
$$

where $\varepsilon=\left[\varepsilon_{r}, \varepsilon_{1}, \ldots, \varepsilon_{2 n}\right]^{T} \in R^{2 n+1}, K_{w} \in R^{2 n+1 \times 2 n+1}$ is a diagonal matrix with $\kappa_{w r}$ as its first diagonal element and $\kappa_{w i}$ as its $(i+1)$ th diagonal element and $K \in R^{2 n+1 \times 2 n+1}$ is a diagonal matrix with $\kappa_{r}$ as its first diagonal element and $\kappa_{i}$ as its $(i+1)$ th diagonal element. It is clear that by choosing the adaptation laws

$$
\begin{array}{r}
\dot{\hat{W}}_{r}=-\Gamma_{r}\left(S_{r}\left(Z_{r}\right) r+\sigma_{r} \hat{W}_{r}\right) \\
\dot{\hat{W}}_{i}=\Gamma_{i}\left(S_{i}\left(Z_{i}\right) z_{i}-\sigma_{i} \hat{W}_{i}\right)
\end{array}
$$

where the second term on the RHS imposes a growth condition on the weight vector with $\sigma_{i}>0$, the following is obtained

$$
\begin{aligned}
\dot{V}< & -z^{T} K z+\frac{1}{4} \bar{\varepsilon}^{T} K_{w}^{-1} \bar{\varepsilon}-\sigma_{r} \tilde{W}_{r}^{T}\left(\tilde{W}_{r}+W_{r}^{*}\right) \\
& -\sum_{i=1}^{2 n} \sigma_{i} \tilde{W}_{i}^{T}\left(\tilde{W}_{i}+W_{i}^{*}\right) \\
< & -z^{T} K z-\frac{\sigma_{r}}{2}\left\|\tilde{W}_{r}\right\|^{2}-\sum_{i=1}^{2 n} \frac{\sigma_{i}}{2}\left\|\tilde{W}_{i}\right\|^{2}
\end{aligned}
$$

$$
\begin{aligned}
& +\frac{1}{4} \bar{\varepsilon}^{T} K_{w}^{-1} \bar{\varepsilon}+\frac{\sigma_{r}}{2}\left\|W_{r}^{*}\right\|^{2}+\sum_{i=1}^{2 n} \frac{\sigma_{i}}{2}\left\|W_{i}^{*}\right\|^{2} \\
< & -\rho V+C
\end{aligned}
$$

where $C:=\frac{1}{2} \bar{\xi}^{T} K_{w}^{-1} \bar{\varepsilon}+\frac{\sigma_{r}}{2}\left\|W_{r}^{*}\right\|^{2}+\sum_{i=1}^{2 n} \frac{\sigma_{i}}{2}\left\|W_{i}^{*}\right\|^{2}$, and

$$
\rho:=\min \left(\frac{2 \lambda_{\min }(K)}{\lambda_{\max }(M)}, \min _{i=r, 1,2, \ldots, 2 n}\left(\frac{\sigma_{i}}{\lambda_{\max }\left(\Gamma_{i}\right)}\right)\right)
$$

and $\lambda_{\min }(\bullet)$ and $\lambda_{\max }(\bullet)$ denote the minimum and maximum eigenvalues of $\bullet$ respectively.

Theorem 3.I: Consider the powertrain dynamics (1) under Assumption 2.1, with control law (6) and adaptation law (9). Given that the initial conditions are bounded, and that full state information is available, the closed loop system is semiglobally uniformly bounded. The closed-loop error signals $z=\left[r, z_{1}, \cdots, z_{2 n}\right]^{T}, \tilde{W}_{r}, \tilde{W}_{i}$ will remain within the compact sets $\Sigma_{z}, \Sigma_{W_{r}}$ and $\Sigma_{W_{i}}$ respectively, defined by

$$
\begin{aligned}
\Sigma_{z} & :=\left\{z \in R^{2 n+1} \mid\|z\| \leq \sqrt{\frac{D}{\lambda_{\min }(M)}}\right\} \\
\Sigma_{W_{r}} & :=\left\{\tilde{W}_{r} \in R^{l_{r}} \mid\left\|\tilde{W}_{r}\right\| \leq \sqrt{\frac{D}{\lambda_{\min }\left(\Gamma_{r}^{-1}\right)}}\right\} \\
\Sigma_{W_{i}} & :=\left\{\tilde{W}_{i} \in R^{l_{i}} \mid\left\|\tilde{W}_{i}\right\| \leq \sqrt{\frac{D}{\lambda_{\min }\left(\Gamma_{i}^{-1}\right)}}\right\}
\end{aligned}
$$

where $D=2\left(V(0)+\frac{C}{\rho}\right)$ with $C$ and $\rho$ as defined in (10) Proof. From (10) and Lemma 2.1, it is straightforward to show that the signals $z, \tilde{W}_{r}, \tilde{W}_{1}, \ldots, \tilde{W}_{2 n}$ are semiglobally uniformly bounded. For completeness, the details of the proof are provided here. Multiplying (10) by $e^{\rho t}$, yields

$$
\frac{d}{d t}\left(V e^{\rho t}\right) \leq C e^{\rho t}
$$

Integrating the above inequality, we obtain

$$
V \leq\left(V(0)-\frac{C}{\rho}\right) e^{-\rho t}+\frac{C}{\rho} \leq V(0)+\frac{C}{\rho}
$$

Substituting (7) into (11), we obtain

$$
\frac{1}{2} \lambda_{\min }(M)\|z\|^{2} \leq V(0)+\frac{C}{\rho}
$$

The bounds of $\left\|\tilde{W}_{r}\right\|$ and $\left\|\tilde{W}_{i}\right\|$ can be similarly shown. This concludes the proof.

\section{Simulation}

In this section, simulation studies are carried out to illustrate the proposed state feedback controller.

Consider a system described below

$$
\begin{aligned}
I \ddot{q} & =k\left(\theta_{1}-q\right)+f(q, \dot{q}) \\
I_{1} \ddot{\theta}_{1} & =k_{1}\left(\theta_{2}-\theta_{1}\right)-k\left(\theta_{1}-q\right)+f_{1}\left(\theta_{1}, \dot{\theta}_{1}\right) \\
I_{2} \ddot{\theta}_{2} & =u-k_{1}\left(\theta_{2}-\theta_{1}\right)+f_{2}\left(\theta_{2}, \dot{\theta}_{2}\right)
\end{aligned}
$$

where $f=-0.16 \dot{q}|\dot{q}|$ is a function of unknown hydrodynamic forces acting on the propeller, $f_{1}=-0.01\left(\dot{\theta}_{1}-\dot{\theta}_{1}\left|\dot{\theta}_{1}\right|\right)$ 
and $f_{2}=-0.01\left(\dot{\theta}_{2}-\dot{\theta}_{2}\left|\dot{\theta}_{2}\right|\right)$ are functions of unknown frictional forces acting on the first and second unit of the shafting system respectively. The torsional stiffness are given by $k=1.5 \times 10^{6}$ and $k_{1}=1.2 \times 10^{6}$. The moment of inertia of the mass units about the rotating axis are given by $I=I_{1}=I_{2}=10$. The desired trajectory is given by

$q_{d}(t)=80 t^{7}-233 t^{9}+305 t^{11}-215 t^{13}+80 t^{15}-12 t^{17}$

and the NNs inputs are as follows:

$$
\begin{aligned}
& Z_{r}=\left[\frac{\ddot{q}_{d}+\lambda \dot{e}}{\epsilon_{1}}, q, \dot{q}\right]^{T} \\
& Z_{1}=\left[q, \dot{q}, \theta_{1}, \frac{1}{\epsilon_{2}} \frac{\partial \alpha_{r}}{\partial \dot{q}}\right]^{T} \\
& Z_{2}=\left[q, \dot{q}, \theta_{1}, \dot{\theta}_{1}, \frac{1}{\epsilon_{3}} \frac{\partial \alpha_{1}}{\partial \dot{q}}, \frac{\phi_{1}}{\epsilon_{4}}\right]^{T} \\
& Z_{3}=\left[q, \dot{q}, \theta_{1}, \dot{\theta}_{1}, \theta_{2}, \frac{1}{\epsilon_{5}} \frac{\partial \alpha_{2}}{\partial \dot{q}}, \frac{1}{\epsilon_{6}} \frac{\partial \alpha_{2}}{\partial \dot{\theta}_{1}}\right]^{T} \\
& Z_{4}=\left[q, \dot{q}, \theta_{1}, \dot{\theta}_{1}, \theta_{2}, \dot{\theta}_{2}, \frac{1}{\epsilon_{7}} \frac{\partial \alpha_{3}}{\partial \dot{q}}, \frac{1}{\epsilon_{8}} \frac{\partial \alpha_{3}}{\partial \dot{\theta}_{1}}, \frac{\phi_{3}}{\epsilon_{9}}\right]^{T}
\end{aligned}
$$

where $\epsilon_{i}$ are design parameters chosen such that the inputs of the NNs are normalized to similar orders of magnitude in the input space in order to facilitate the placement of nodes and improve the performance of the NNs.

In the following simulation study, we select the centers and widths of the RBF's as: Neural Network $\hat{W}_{r}^{T} S_{r}$ contains 8 nodes (i.e., $l_{r}=8$ ), with centers $\mu_{\tau l}\left(l=1, \ldots, l_{r}\right)$ evenly spaced in $[-5,10] \times[-5,15] \times[-10,10]$, and widths $\eta_{r l}^{2}=20\left(l=1, \ldots, l_{r}\right)$. Neural Network $\hat{W}_{1}^{T} S_{1}$ contains 16 nodes (i.e., $l_{1}=16$ ), with centers $\mu_{1 l}\left(l=1, \ldots, l_{1}\right)$ evenly spaced in $[-5,15] \times[-10,10] \times[-5,15] \times[-10,10]$, and widths $\eta_{1 l}^{2}=100\left(l=1, \ldots, l_{2}\right)$. Neural Network $\hat{W}_{2}^{T} S_{2}$ contains 64 nodes (i.e., $l_{2}=64$ ), with centers $\mu_{2 l}\left(l=1, \ldots, l_{2}\right)$ evenly spaced in $[-5,15] \times[-10,10] \times$ $[-5,15] \times[-10,10] \times[-10,10] \times[-10,10]$, and widths $\eta_{2 l}^{2}=300\left(l=1, \ldots, l_{3}\right)$. Neural Network $\hat{W}_{3}^{T} S_{3}$ contains 128 nodes (i.e., $l_{3}=128$ ), with centers $\mu_{3 l}\left(l=1, \ldots, l_{3}\right.$ ) evenly spaced in $[-5,15] \times[-10,10] \times[-5,15] \times[-10,10] \times$ $[-5,15] \times[-10,10] \times[-10,10]$, and widths $\eta_{3 l}^{2}=500(l=$ $\left.1, \ldots, l_{3}\right)$. Neural Network $\hat{W}_{4}^{T} S_{4}$ contains 512 nodes (i.e., $\left.l_{4}=512\right)$, with centers $\mu_{4 l}\left(l=1, \ldots, l_{4}\right)$ evenly spaced in $[-5,15] \times[-10,10] \times[-5,15] \times[-10,10] \times[-5,15] \times$ $[-10,10] \times[-10,10] \times[-10,10] \times[-10,10]$, and widths $\eta_{4 l}^{2}=700\left(l=1, \ldots, l_{4}\right)$. The initial weight estimates are set to be 0 , i.e., $\hat{W}_{r}(0)=0, \hat{W}_{1}(0)=0, \hat{W}_{2}(0)=0, \hat{W}_{3}(0)=0$ and $\hat{W}_{4}(0)=0$..

The following initial conditions and controller design parameters are adopted in the simulation: $x(0)=$ $[0,0,0.005,0,0,0]^{T}, \Gamma_{r}=\operatorname{diag}\{65\}, \Gamma_{1}=\operatorname{diag}\{15\}$, $\Gamma_{2}=\operatorname{diag}\{45\}, \Gamma_{3}=\operatorname{diag}\{15\}, \Gamma_{4}=\operatorname{diag}\{20\}, \sigma_{r}=$ $\sigma_{1}=\sigma_{2}=\sigma_{3}=0.1, \sigma_{4}=0.01, \kappa_{r}=\kappa_{w r}=1.5$, $\kappa_{1}=\kappa_{w 1}=6, \kappa_{2}=\kappa_{w 2}=0.00005, \kappa_{3}=\kappa_{w 3}=2.5$, $\kappa_{4}=\kappa_{w 4}=0.00005, \lambda=12, \epsilon_{1}=5, \epsilon_{2}=0.8, \epsilon_{3}=2$, $\epsilon_{4}=80, \epsilon_{5}=2, \epsilon_{6}=0.5, \epsilon_{7}=60, \epsilon_{8}=25$ and $\epsilon_{9}=7000$.
Fig. 1 shows that tracking of the desired propeller velocity is achieved. Figs. 2 and 3 shows that torsional vibrations within the shafting system, due to the initial torsional displacements $q(0)-\theta_{1}(0)=-0.005$ and $\theta_{1}(0)-\theta_{2}(0)=0.005$, is reduced after 1 second. However, it is noted that, under the proposed control law, the torsional vibrations within the shafting system does not converge to zero. This problem may be reduced through a proper selection of the design parameters and further studies are to be conducted in order to improve the performance of the proposed controller. Figs. 4,5 and 6 show the boundedness of the control input and the NN weights in the control loop.

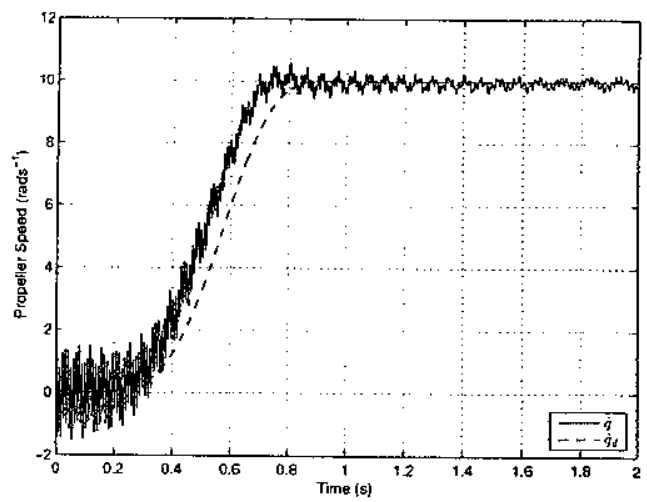

Fig. 1. Propeller velocity, $\dot{q}$, and desired velocity

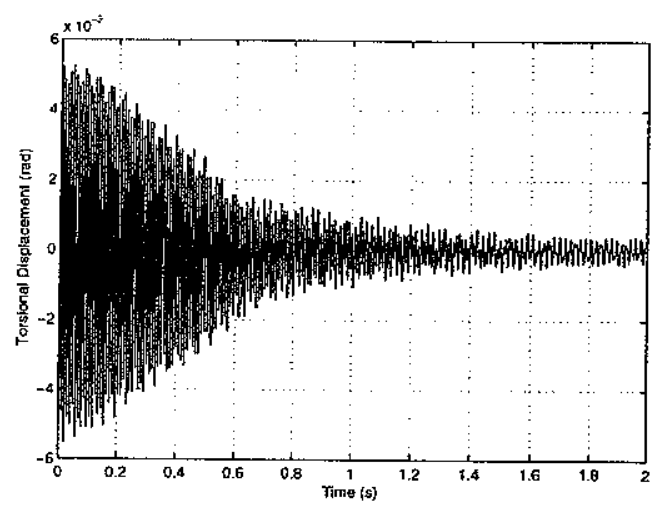

Fig. 2. Torsional Displacement $q-\theta_{1}$

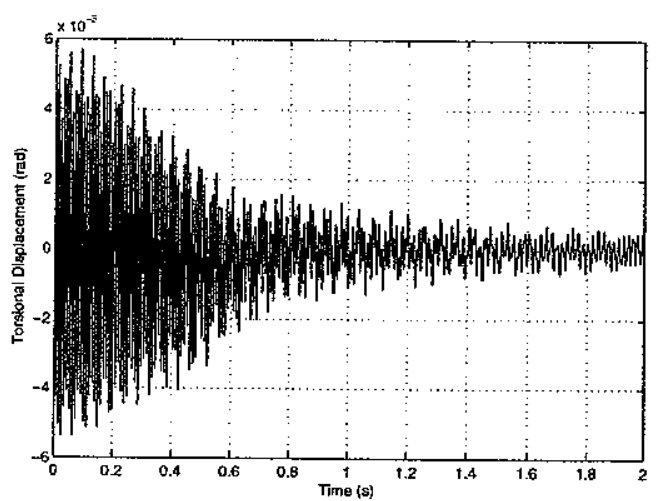

Fig. 3. Torsional Displacement $\theta_{1}-\theta_{2}$ 


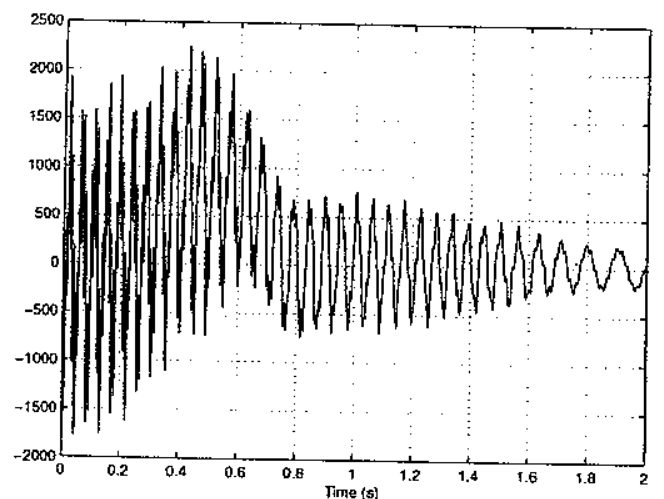

Fig. 4. Control lnput $u(t)$

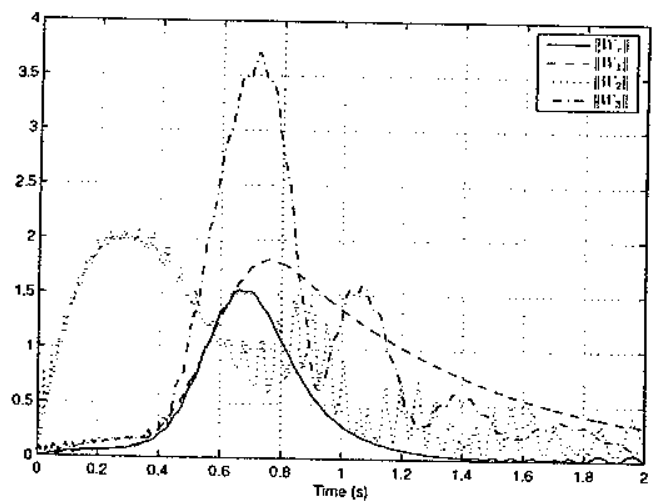

Fig. 5. Norms of NN weights $\left\|\hat{W}_{r}\right\|,\left\|\hat{W}_{1}\right\|,\left\|\hat{W}_{2}\right\|$ and $\left\|\hat{W}_{3}\right\|$

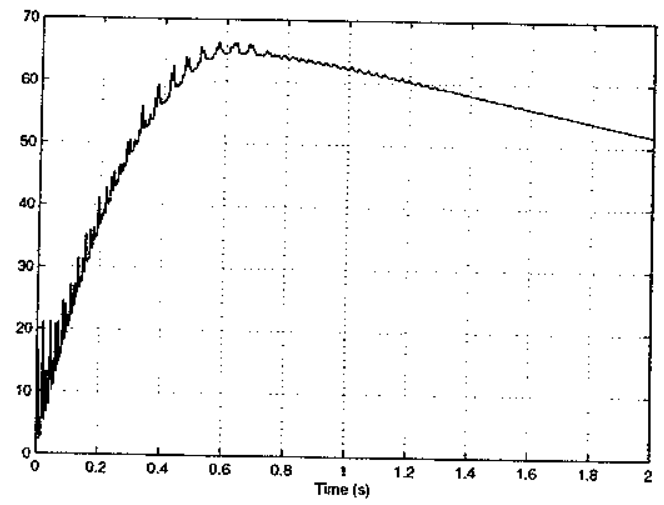

Fig. 6. Norms of NN weight $\left\|\hat{W}_{A}\right\|$

\section{CONCLUSION}

In this paper, an approximation-based control design is developed with the objective of solving a marine propulsion control problem while simultaneously suppressing the torsional vibrations within the shafting system, in the presence of unmodelled dynamics, or parametric/functional uncertainties. The controller is mathematically shown to guarantee semiglobally uniformly bounded stability, and the steady state compact set to which the closed loop error signals converge is derived. The size of compact set can be made small through appropriate choice of the control design parameters.
Simulation results demonstrate that the system, under the proposed control and adaptation Jaws, is able to achieve the desired shaft speed and the torsional vibrations within the shafting system are reduced, with all closed loop signals uniformly bounded. Although the torsional vibrations are noticeably reduced under the proposed control law, it does not converge to zero as illustrated in the simulation results. Further studies are to be conducted in order to improve the performance of the proposed controller.

\section{REFERENCES}

[1] W. K. Wilson, Practical solution of torsional vibration problems. London: Chapman \& Hall, 1971.

[2] J. Wu and W. Chen, "Computer method for forced torsional vibration of propulsive shafting system of marine engine with or without damping," Journal of Ship Research. vol. 26, no. 3, pp. 176-189, 1982.

[3] K. Bruun, E. Pedersen. and H. Valiand, "The development of vehicular powertrain system modeling methodologies: Philosophy and implementation," in Proceedings of the Imternational Conference on Bond Graph Modeling, 2005.

[4] T. 1. Fossen and M. Blanke. "Nonlinear output feedback control of underwater vehicle propellers using feedback form estimated axial flow velocity," IEEE Journal of Oceanic Engineering, vol. 25, no. 2, pp. $241-255,2000$

[5] A. J. Sørensen, A. K. Ådnanes. T. I. Fossen, and J. P. Strand, "A new method of thruster control in positioning of ships based on power control," in Proceedings of the IFAC Conference on Manoeuvering and Control of Marine Craft. Brijuni, Croatia, Sept 1997, pp. 172179.

[6] J. P. Breslin and P. Andersen, Hydrodrnamics of Ship Propellers. Cambridge: University Press, $1994^{\circ}$

[7] R. M. Sanner and J. E. Slotine. "Gaussian networks for direct adaptive control," IEEE Transactions on Neural Networks, vol. 3, no. 6, pp. $837-863,1992$

[8] S. S. Ge, "Advanced control techniques of robotic manipulators," in Proceedings of the American Control Conference, Philadelphia, Pennsylvania, Jun 1998, pp. 2185-2199.

[9] S. S. Ge, T. H. Lee, and C. J. Harris, Adaptive Neural Network Control of Robotic Manipulators. London: World Scientific, 1998.

[10] C. M. Kwan, F. L. Lewis, and Y. H. Kim. "Robust neural network control of flexible-joint robots," in Proceedings of the 34th Conference on Decision \& Control, New Orleans. Louisiana, Dec 1995, pp. 12961301.

[11] S. S. Ge and C. Wang, "Adaptive neural control of uncertain mimo nonlinear systems," IEEE Transactions on Neural Nerworks, vol. 15, no. 3 , pp. $674-692,2004$

[12] S. Haykin, Neural Networks - A Comprehensive Foundation. USA: Macmillan College, 1994

[13] S. S. Ge, C. C. Hang. T. H. Lee, and T. Zhang, Stable Adaptive Neural Network Control. Norwell, USA: KJuwer Academic, 2001.

[14] M. Krstić, I. Kanellakopoulos, and P. V. Kokotovic, Nonlinear and Adaptive Control Design. New York: Wiley, 1995.

[15] J. E. Slotine and W. Li, Nonlinear Control. Englewood Cliff, NJ: Prentice-Hall, 1991.

[16] L. L. Whitcomb and D. R. Yoerger, "Development, comparison, and preliminary experimental validation of nonlinear dynamic thruster models," IEEE Journal of Oceanic Engineering, vol. 24, no. 4, pp. $481-494,1999$.

[17] _-., "Development, comparison, and preliminary experimental validation of nonlinear dynamic thruster models," IEEE Journal of Oceanic Engineering, vol. 24, no. 4, pp. 495-506, 1999.

[18] J. Wu and M. Hsieh, "Torsional vibration of a damped shaft system using the analytical-and-numerical-combined method," Marine Technology, vol. 38 , no. 4 , pp. $250-260,2001$. 